\title{
Correspondence between dark energy quantum cosmology and Maxwell equations
}

\author{
Felipe A. Asenjo ${ }^{1, \mathrm{a}}$, Sergio A. Hojman ${ }^{2,3,4, b}$ \\ ${ }^{1}$ Facultad de Ingeniería y Ciencias, Universidad Adolfo Ibáñez, 7941169 Santiago, Chile \\ ${ }^{2}$ Departamento de Ciencias, Facultad de Artes Liberales, Universidad Adolfo Ibáñez, Santiago, Chile \\ ${ }^{3}$ Departamento de Física, Facultad de Ciencias, Universidad de Chile, Santiago, Chile \\ ${ }^{4}$ Centro de Recursos Educativos Avanzados, CREA, Santiago, Chile
}

Received: 14 May 2019 / Accepted: 27 August 2019 / Published online: 4 September 2019

(C) The Author(s) 2019, corrected publication 2019

\begin{abstract}
A Friedmann-Robertson-Walker cosmology with dark energy can be modelled using a quintessence field. That system is equivalent to a relativistic particle moving on a two-dimensional conformal spacetime. When the quintessence behaves as a free massless scalar field in a Universe with cosmological constant, the quantized version of that theory can lead to a supersymmetric Majorana quantum cosmology. The purpose of this work is to show that such quantum cosmological model corresponds to the Maxwell equations for electromagnetic waves propagating in a medium with specific values for its relative permittivity and relative permeability. The form of those media parameters are calculated, implying that a Majorana quantum cosmology can be studied in an analogue electromagnetic system.
\end{abstract}

\section{Introduction}

The aim of this work is to show that there exists a correspondence between the seemingly different physical models of a cosmological model using dark energy and Maxwell equations. The link between these two formalisms appears when one considers the quantum version of the cosmological model [1] using the Breit prescription [2] for spin particles.

The representation of dark energy using a quintessence field with a potential, allows us to describe the cosmological dynamics in fashion which is analogous to the description of the dynamics of a relativistic particle. In the case of quintessence described by a free massless scalar field in a Universe with cosmological constant, the resultant theory may be quantized by using a Klein-Gordon scheme, giving

\footnotetext{
a e-mail: felipe.asenjo@uai.cl

b e-mail: sergio.hojman@uai.cl
}

rise to the Wheeler-DeWitt equation [3-13]. However, using the Breit prescription [2], the same model can be quantized as a spinorial theory. This procedure yields a Majorana version for the quantum cosmology which happens to be supersymmetric [1].

In addition of the cosmological implications of such theory, the aim of this article is to show its direct correspondence with the description of propagating electromagnetic fields in a medium using Maxwell equations. We can identify the relative permittivity and permeability of the medium with parameters of the quantum cosmological model. As we show, this implies that the supersymmetric Majorana quantum cosmology can be studied in an analogue electromagnetic system using either normal materials or negative-index metamaterials (NIMs) [14,15].

\section{Quantum cosmology with dark energy}

Consider an isotropic and homogeneous FriedmannRobertson-Walker (FRW) spacetime with a line element [16]

$d s^{2}=d t^{2}-a(t)^{2}\left[\frac{d r^{2}}{1-k r^{2}}+r^{2}\left(d \theta^{2}+\sin ^{2} \theta d \phi^{2}\right)\right]$,

where $a(t)$ is the scale factor, and the curvature constant $k=$ $\pm 1,0$. The evolution of a FRW cosmology with cosmological constant $\Lambda$, interacting with a quintessence (massless scalar) field $\phi\left(x^{\beta}\right)$ characterized by a potential $\mathcal{V}(\phi)$, can be found by using Einstein equations [1] $\left(8 \pi G / c^{4}=1\right.$, where $G$ is the gravitational constant and $c$ is the speed of light)

$$
\begin{aligned}
2 \frac{\ddot{a}}{a}+\left(\frac{\dot{a}}{a}\right)^{2}+\frac{k}{a^{2}}+\frac{1}{2} \dot{\phi}^{2}-V(\phi) & =0, \\
3\left(\frac{\dot{a}}{a}\right)^{2}+3 \frac{k}{a^{2}}-\left(\frac{1}{2} \dot{\phi}^{2}+V(\phi)\right) & =0,
\end{aligned}
$$


where we introduced $V(\phi)=\mathcal{V}(\phi)-\Lambda$. Also, the KleinGordon equation for the quintessence field is

$\ddot{\phi}+3 \frac{\dot{a}}{a} \dot{\phi}+\frac{d V(\phi)}{d \phi}=0$.

The above system describes the evolution of a Friedmann-

Robertson-Walker-Quintessence (FRWQ) Universe.

In Ref. [1] was shown that the Lagrangian

$L_{F}=\sqrt{\bar{V}(\xi, \theta) e^{2 \xi}\left(\dot{\theta}^{2}-\dot{\xi}^{2}\right)}$,

gives rise to all three equations (2)-(4), with variables $\xi=$ $\ln \left(2 \sqrt{6} a^{3 / 2} / 3\right), \theta=3 \phi /(2 \sqrt{6})$ [here $\dot{\xi}=d \xi / d \lambda$ and $\dot{\theta}=$ $d \theta / d \lambda$, with $\lambda$ is an arbitrary parameter], and the general potential

$\bar{V}(\xi, \theta)=3\left(\frac{3}{8}\right)^{1 / 3} k e^{2 \xi / 3}-\left(\frac{3}{8}\right) e^{2 \xi} V(\theta)$.

This Lagrangian (5) shows that the FRWQ cosmology evolves as a relativistic particle moving in two dimensional spacetime under the influence of potential $\bar{V}$. It can be proved that the Lagrangian (5) gives rise to the FRWQ equations by recalling that the Jacobi-Maupertuis and Fermat principles [17] yield identical equations of motion in classical mechanics and geometrical (ray) optics except for the fact that Fermat principle also produces a constraint equation. Notice that Lagrangian (5) can be written as the one for a relativistic particle in a two-dimensional conformally flat spacetime

$L_{F}=\sqrt{g_{\mu \nu}\left(\frac{d x^{\mu}}{d \lambda}\right)\left(\frac{d x^{\nu}}{d \lambda}\right)}$,

with the metric $g_{\mu \nu}=\Omega^{2} \eta_{\mu \nu}$ (where $\eta_{\mu \nu}$ is the flat spacetime metric, and $x^{0}=\theta$ and $x^{1}=\xi$ ), and the conformal factor

$\Omega \equiv \sqrt{\bar{V}} e^{\xi}=\left[3\left(\frac{3}{8}\right)^{\frac{1}{3}} k e^{8 \xi / 3}-\frac{3}{8} V(\theta) e^{4 \xi}\right]^{1 / 2}$,

Thus, the FRWQ system is equivalent to a relativistic particle moving in a two-dimensional conformally flat spacetime, where the quintessence field plays the role of an effective time.

The above results allow to find the quantum version of the FRWQ cosmology, through quantization of the Lagrangian (5). We restrict ourselves to cases where $\bar{V}>0$, in order to avoid problems in the quantization procedure. We also consider static manifolds [18] such that there exists a family of spacelike surfaces orthogonal to a timelike Killing vector. Therefore, Lagrangian (5) is always real, which can be proved by using the definitions of $\xi, \theta$ and $\bar{V}$. All of this implies that $\partial_{\theta} g_{\mu \nu}=0$, or $\partial_{\theta} \bar{V}=0$, describing a constant potential $V(\theta)$ (becoming essentially equal to a cosmological constant). Thus, the associated Noether conservation law implies that $\dot{\theta}$ does not change sign, and $\theta$ can be used as the evolution time variable. Using this $\theta$-time, it can be shown at classical level [19] that the Hamiltonian for Lagrangian (5) is $H=\sqrt{g_{00}} \sqrt{1-g^{11} \pi^{2}}=\sqrt{g_{00}} \sqrt{1+\pi^{2} / \Omega^{2}}$, where $\pi$ is the canonical momentum, and $\sqrt{g_{00}}=\Omega$. This classical Hamiltonian is used to construct the quantum Hamiltonian operator $\mathcal{H}$ as [1]

$\mathcal{H}=\Omega^{1 / 2} \sqrt{1+\hat{p}^{2}} \Omega^{1 / 2}, \quad \hat{p}=\sqrt{-g^{11}} \hat{\pi}=-\frac{i}{\Omega} \frac{\partial}{\partial \xi}$,

where $\hat{p}$ is the momentum operator and $\hat{\pi}=-i \partial_{\xi}$. Therefore, the quantum equation that describes the quantization of the FRWQ system is

$i \hbar \frac{\partial \Psi}{\partial \theta}=\mathcal{H} \Psi$,

where $\Psi$ is the wavefunction for the quantum FRWQ cosmology. The quantum equation (10) emerges because of the direct correspondence between the FRW geometry and the quintessence scalar field at a Fermat-like Lagrangian level. To find a solution, we need a quantization procedure to solve the square-root of the Hamiltonian operator (9). It is costumary to solve the square-root using a spinless particle approach to get a Klein-Gordon equation [20], which gives origin to the Wheeler-DeWitt super-Hamiltonian formalism. However, notice that there is no restriction for the quantization scheme possible to be used. In principle, we can solve the square-root using matrices, obtaining the quantization of a relativistic particle which leads to the Dirac equation. This procedure was introduced by Breit [2], that shows that there is a correspondence between the Dirac and the relativistic pointlike particle Hamiltonians. Breit's interpretation [2] identifies the Dirac matrices as $\pi / H \rightarrow \alpha$, and $\sqrt{1-(\pi / H)^{2}} \rightarrow \beta$. These identifications are consistent with the postulates of Dirac electron's theory $[2,21,22]$. By the Breit's prescription, the Hamiltonian operator (9) becomes [1]

$\mathcal{H}=\Omega^{1 / 2}(\alpha \cdot \hat{p}+\beta) \Omega^{1 / 2}$,

where $\alpha$ and $\beta$ are the two-dimensional flat spacetime Dirac matrices, as the effective curvature is already taken into account in $\Omega$ (with $\hbar=1$ ). Moreover, now the wavefunction $\Psi$ [in Eq. (10)] is a two-dimensional spinor.

With this Hamiltonian, and defining the wavefunction $\Phi=\sqrt{\Omega} \Psi$, we finally find from Eq. (10) the spinor quantum equation $[1,23]$

$i \gamma^{0} \frac{\partial \Phi}{\partial \theta}+i \gamma^{1} \frac{\partial \Phi}{\partial \xi}=\Omega \Phi$.

where $\gamma^{0}=\beta$ and $\gamma^{1}=\gamma^{0} \alpha$. The above equation corresponds to a Quantum Cosmology theory for the FRWQ system, modelling now the Universe as a spin particle in a two-dimensional conformally flat spacetime [1]. 
In order to obtain real wavefunctions $\Phi$, the matrices in Eq. (12) should correspond to the two-dimensional Majorana representation

$\gamma^{0}=\left(\begin{array}{cc}0 & -i \\ i & 0\end{array}\right), \quad \gamma^{1}=\left(\begin{array}{cc}i & 0 \\ 0 & -i\end{array}\right)$.

In this form, Eq. (12) becomes a set of supersymmetric equations of quantum mechanics [1,24-28], which can only be obtained in the Majorana picture. The implications of this system were thoroughly studied in Ref. [1], showing that Eq. (12) with matrices (13) represents a Supersymmetric Majorana quantum cosmology. This can be seen by defining

$\Phi(\theta, \xi)=\left(\begin{array}{c}\varphi_{+}(\xi) e^{E \theta} \\ \varphi_{-}(\xi) e^{-E \theta}\end{array}\right)$,

to find from (12) the set of supersymmetric equations of quantum mechanics [1,23-28]

$Q_{ \pm} \varphi_{ \pm}=E \varphi_{\mp}$.

with the operators $Q_{ \pm}= \pm d_{\xi}+\Omega$. These equations are supersymmetric with the two spinor components being super-partners of each other. Each wavefunction satisfies $H_{ \pm} \varphi_{ \pm}=E^{2} \varphi_{ \pm}$, with the Hamiltonians operators $H_{ \pm}=$ $-d_{\xi}^{2}+\mathcal{W}_{ \pm}$, and potentials $\mathcal{W}_{ \pm}=\mp d_{\xi} \Omega+\Omega^{2}$ [1]. Also, $Q_{ \pm}$correspond to supercharge operators. This theory can produce new versions of quantum cosmological models. For example, it can be shown that in flat curvature case, the Universe behaves as a diatomic molecule subject to the Morse potential [1].

\section{Correspondence to Maxwell equations}

In this section we focus the correspondence between the theory (12) and electromagnetism. In order to make this correspondence manifest, let us consider Maxwell equations in a medium with permittivity $\epsilon$ and permeability $\mu$ in the absence of charges [29]

$$
\begin{aligned}
& \frac{\partial \mathbf{D}}{\partial t}=\nabla \times \mathbf{H}, \\
& \frac{\partial \mathbf{B}}{\partial t}=-\nabla \times \mathbf{E},
\end{aligned}
$$

with the electric field $\mathbf{E}$, the magnetic field $\mathbf{B}$, the displacement field $\mathbf{D}=\epsilon \mathbf{E}$, the magnetization field $\mathbf{H}=\mathbf{B} / \mu$ (chosing the speed of light $c=1$ ). Furthermore, $\nabla \cdot \mathbf{D}=0$ and $\nabla \cdot \mathbf{B}=0$.

In order to show the correspondence with the Quantum Cosmology model, we assume that the permittivity and the permeability are not constant. Also, let us consider a twodimensional spacetime system (one temporal and one spatial dimension), with spatial variations in, let us say, the $\hat{e}_{z^{-}}$ direction. We choose transverse fields $\mathbf{B}(t, z)=B(t, z) \hat{e}_{x}$, and $\mathbf{D}(t, z)=D(t, z) \hat{e}_{y}$, such that $\mathbf{B} \cdot \hat{e}_{z}=0=\mathbf{D} \cdot \hat{e}_{z}$, and $\mathbf{B} \cdot \mathbf{D}=0$. Besides, the time-independent permittivity and permeability have the same spatial dependence, $\epsilon=\epsilon(z)$ and $\mu=\mu(z)$. Then, Maxwell equations (16) acquire the form

$$
\begin{aligned}
& \frac{\partial \mathcal{D}}{\partial t}=-\frac{B}{\hat{\mu}^{2}} \frac{\partial \hat{\mu}}{\partial z}+\frac{1}{\hat{\mu}} \frac{\partial B}{\partial z}, \\
& \frac{\partial B}{\partial t}=-\frac{\mathcal{D}}{\hat{\epsilon}^{2}} \frac{\partial \hat{\epsilon}}{\partial z}+\frac{1}{\hat{\epsilon}} \frac{\partial \mathcal{D}}{\partial z} .
\end{aligned}
$$

where $\mathcal{D}=\mu_{0} D$, the relative permittivity is $\hat{\epsilon}=\epsilon / \epsilon_{0}$, the relative permeability is $\hat{\mu}=\mu / \mu_{0}$, and $\epsilon_{0}$ and $\mu_{0}$ are the free-space permittivity and permeability respectively (with $\left.\epsilon_{0} \mu_{0}=1\right)$.

By introducing the spinor

$\Phi=\left(\begin{array}{l}B \\ \mathcal{D}\end{array}\right)$

we can rewrite system (17) in a simple way as

$i \gamma^{0} \frac{\partial \Phi}{\partial t}+i \Gamma_{1} \frac{\partial \Phi}{\partial z}=\Gamma_{2} \Phi$

with $\gamma^{0}$ given in (13), and the matrices

$\Gamma_{1}=\left(\begin{array}{cc}i / \hat{\mu} & 0 \\ 0 & -i / \hat{\epsilon}\end{array}\right), \quad \Gamma_{2}=\left(\begin{array}{cc}-\hat{\mu}^{\prime} / \hat{\mu}^{2} & 0 \\ 0 & \hat{\epsilon}^{\prime} / \hat{\epsilon}^{2}\end{array}\right)$,

with $\hat{\mu}^{\prime}=\partial \hat{\mu} / \partial z$ and $\hat{\epsilon}^{\prime}=\partial \hat{\epsilon} / \partial z$. The spinor form (19) of Maxwell equations is general for a two-dimensional spacetime. For any general permittivity and permeability, Eq. (19) does not coincide with the Quantum cosmology equation (12). For example, for vacuum, $\Gamma_{2}=0$.

However, we can show that there exists a regime in which Maxwell equations and the Supersymmetric Majorana Quantum Cosmology coincide. Let us consider the following form for the relative permittivity and relative permeability

$\hat{\epsilon}(z) \approx \pm 1+\lambda(z), \quad \hat{\mu}(z) \approx \pm 1-\lambda(z)$,

in terms of a function $\lambda$ to be determined. We focus our attention in the regime when $|\lambda| \ll 1$. The relative permeability and permittivity of this material are almost equal, $\sqrt{\hat{\mu} / \hat{\epsilon}} \approx 1-\lambda$. With the relative permittivity and permeability given by (21), the matrices (20) becomes

$\Gamma_{1} \approx \pm\left(1+\lambda^{2}\right) \gamma^{1} \pm i \lambda \mathbf{1}$

$\Gamma_{2} \approx \frac{d \lambda}{d z} \mathbf{1} \mp i \frac{d \lambda^{2}}{d z} \gamma^{1}$

with the matrix $\gamma^{1}$ given by (13), and the two-dimensional unit matrix 1. In general, for any $\hat{\epsilon}$ and $\hat{\mu}$, Eq. (19) does not have a conserved current $\bar{\Phi} \gamma^{\mu} \Phi=\Phi^{\dagger} \Phi+\Phi^{\dagger} \gamma^{0} \gamma^{1} \Phi$. Nonetheless, for materials satisfying (21) and (22), Eq. (19) can have a conserved current if $|\lambda| \ll 1$ and that the variation ranges of $B$ and $D$ are much larger than the variation range 
of $\lambda$, i.e., $\lambda(\partial \Phi / \partial z) \ll(d \lambda / d z) \Phi$. Using these approximations, Maxwell equation (19) can be written in an approximated form as

$i \gamma^{0} \frac{\partial \Phi}{\partial t}+i \gamma^{1} \frac{\partial \Phi}{\partial z}=\frac{d \lambda}{d z} \Phi$,

which has the usual conservartion law for a Dirac equation. With all of the above, the correspondence between Maxwell equations (23) and the supersymmetric quantum Majorana cosmologies equation (12) is now evident by redefining

$t=\theta$,

$\xi= \pm z$

$\lambda=\lambda(\xi)=\int \Omega d \xi=-\frac{4}{3 V} e^{-4 \xi} \Omega^{3}$.

This last equation implies that this correspondence is only valid in the regime $\Omega^{3} e^{-4 \xi} \ll|V|$, which is the limit needed in order to keep current conservation.

Results (24) establish the complete correspondence bet ween a Supersymmetric Majorana Quantum cosmological model and Maxwel equations. Materials satisfying (21) must have almost equal relative permeability and permittivity, and they can be both positive or both negative. In the former case, we are describing a normal material with such a property. In case that both permeability and permittivity are negative, we are describing a NIM $[14,15,30]$. In general, the refraction index is

$n(\xi)=\sqrt{\hat{\epsilon}(\xi) \hat{\mu}(\xi)} \approx \pm\left(1-\frac{8}{9 V^{2}} e^{-8 \xi} \Omega^{6}\right)$,

where the positive sign describes a normal material, and the negative refractive index represents a NIM in which a wave propagates backwards.

There are materials with relative permittivity and relative permeability that can have the form (21). Composite ferrites [31-34] can, under appropriated conditions, achieve almost matching permeability and permittivity values by shining radiation of different frequencies on the material. This implies that the above results can be tested in an analogue fashion using those materials. For example, for a spatially flat cosmology, the Maxwell equations and the Supersymmetric Majorana quantum cosmology coincide for $\lambda(\xi)=\sqrt{-3 V / 32} \exp (2 \xi)$, with constant $V<0$, and refraction index $n(\xi) \approx \pm[1+3 V \exp (4 \xi) / 64]$. For this case, Maxwell equations can only describe a quantum cosmology for $\xi \ll \ln (11 /|V|)$. Furthermore, the boundary conditions for $\Phi$ given in Eq. (18) (which depend on $\Omega$ ) should be suitable for simulating a quantum cosmology. For $k=0$, the boundary conditions for $\xi \rightarrow \infty(a \rightarrow \infty)$ can be established to obtain a vanishing magnetic field at infinity in one spatial dimension (see Ref. [1]).

Our proposal is in the same spirit than similar ones for analogue optical systems for quantum cosmologies $[35,36]$, and for gravity in general (see for example Refs. [37-40]). However, our result establishes an "optical" analogue for a new kind of spinor quantized cosmological model. The proposed analogue electromagnetic media that correspond to the quantum cosmology is time-independent but space-dependent, which is an approach opposite to previous attempts [35]. Relations (21) are satisfied by certain tunable metamaterials $[41,42]$ and composite ferrites [31-34] used to operate at a wider range of frequencies. All of the above makes of this Majorana Supersymetric quantum cosmological model a system worth to be studied by studying wave propagation in Maxwell equations in the appropriated media.

Data Availability Statement This manuscript has no associated data or the data will not be deposited. [Authors' comment: Data sharing not applicable to this article as no datasets were generated or analysed during the current study.]

Open Access This article is distributed under the terms of the Creative Commons Attribution 4.0 International License (http://creativecomm ons.org/licenses/by/4.0/), which permits unrestricted use, distribution, and reproduction in any medium, provided you give appropriate credit to the original author(s) and the source, provide a link to the Creative Commons license, and indicate if changes were made.

Funded by SCOAP ${ }^{3}$.

\section{References}

1. S.A. Hojman, F.A. Asenjo, Phys. Rev. D 92, 083518 (2015)

2. G. Breit, Proc. Natl. Acad. Sci. 14, 555 (1928)

3. B.S. DeWitt, Phys. Rev. 160, 1113 (1967)

4. J.J. Halliwell, S.W. Hawking, Phys. Rev. D 31, 1777 (1985)

5. F.G. Alvarenga, N.A. Lemos, Gen. Relativ. Gravit. 30, 681 (1998)

6. G. Oliveira-Neto, Phys. Rev. D 58, 107501 (1998)

7. N.A. Lemos, J. Math. Phys. 37, 1449 (1996)

8. G.A. Monerat, E.V. Corrêa Silva, G. Oliveira-Ne, L.G. Ferreira Filho, N.A. Lemos, Phys. Rev. D 73, 044022 (2006)

9. G. Oliveira-Neto, G.A. Monerat, E.V. Corrêa Silva, C. Neves, L.G. Ferreira Filho, Int. J. Theor. Phys 52, 2991 (2013)

10. B. Vakili, Ann. Phys. (Berlin) 19, 359 (2010)

11. R.-N. Huang, (2013). arXiv:1304.5309v2

12. J.B. Barbour, N.'O Murchadha, (1999). arXiv:gr-qc/9911071v1

13. S.W. Hawking, Z.C. Wu, Phys. Lett. 151B, 15 (1985)

14. N. Engheta, R.W. Ziolkowski, Metamaterials: Physics and Engineering Explorations (Wiley, New York, 2006)

15. G.V. Eleftheriades, K.G. Balmain, Negative-Refraction Metamaterials: Fundamental Principles and Applications (Wiley, New York, 2005)

16. B. Ryden, Introduction to Cosmology (Addison Wesley, San Francisco, 2003)

17. S.A. Hojman, S. Chayet, D. Núñez, M.A. Roque, J. Math. Phys. 32, 1491 (1991)

18. A. Saa, Class. Quantum Grav. 13, 553 (1996)

19. A. Hanson, T. Regge, C. Teitelboim, Constrained Hamiltonian Systems (Accademia Nazionale dei Lincei, Rome, 1976)

20. S.P. Gavrilov, D.M. Gitman, Class. Quantum Grav. 17, L133 (2000)

21. S. Savasta, O. Di Stefano, (2008). arXiv:0803.4013v1

22. S. Savasta, O. Di Stefano, O. M. Maragò, (2009). arXiv:0905.4741v1

23. C.A. Rubio, F.A. Asenjo, S.A. Hojman, Symmetry 11, 860 (2019) 
24. P.D. D'Eath, Supersymmetric Quantum Cosmology (Cambridge University Press, Cambridge Monographs on Mathematical Physics, Cambridge, 1996)

25. P. VargasMoniz, Quantum Cosmology-The Supersymmetric Perspective_Vol. 1: Fundamentals, Lecture Notes Physics 803 (Springer, Berlin, 2010)

26. F. Cooper, A. Khare, R. Musto, A. Wipf, Ann. Phys. 187, 1 (1988)

27. M. de Crombrugghe, V. Rittenberg, Ann. Phys. 151, 99 (1983)

28. F. Cooper, A. Khare, U. Sukhatme, Supersymmetry in Quantum Mechanics (World Scientific, Singapore, 2001)

29. J.D. Jackson, Classical Electrodynamics (Wiley, New York, 1975)

30. G.A. Kraftmakher, V.S. Butylkin, Tech. Phys. Lett. 29, 230 (2003)

31. Z. Zheng, H. Zhang, J.Q. Xiao, F. Bai, IEEE Trans. Magn. 49, 4214 (2013)

32. H. Su et al., J. Appl. Phys. 113, 17B301 (2013)

33. L.B. Kong, Z.W. Li, G.Q. Lin, Y.B. Gan, IEEE Trans. Magn. 43, 6 (2007)

34. A. Thakur, P. Thakur, J.-H. Hsu, Scr. Mater. 64, 205 (2011)
35. N. Westerberg et al., New J. Phys. 16, 075003 (2014)

36. A.B. Batista et al., Phys. Rev. D 65, 063519 (2002)

37. M. Visser, C. Barceló, S. Liberati, Gen. Relativ. Gravit. 34, 1719 (2002)

38. C. Barceló, S. Liberati, M. Visser, Living Rev. Relativ. 14, 3 (2011)

39. M. Ornigotti, S. Bar-Ad, A. Szameit, V. Fleurov, Phys. Rev. A 97, $013823(2018)$

40. D. Faccio, F. Belgiorno, S. Cacciatori, V. Gorini, S. Liberati, U. Moschella, Analogue Gravity Phenomenology: Analogue Spacetimes and Horizons, from Theory to Experiments (Springer, Berlin, 2013)

41. K. Bi et al., PLoS One 10, e012733 (2015)

42. P.S. Grant et al., Philos. Trans. R. Soc. A 373, 20140353 (2015) 